\title{
Gasto energético e consumo de oxigênio pós-exercício contra-resistência
}

\author{
Energy expenditure and excess post-exercise \\ oxygen consumption of resistance exercise
}

Cristiane MATSUURA ${ }^{1}$

Cláudia de Mello MEIRELLES $1,2,3$

Paulo Sérgio Chagas GOMES 1

RE S U M O

O aumento do gasto energético com a atividade física é reconhecido como um importante coadjuvante em programas para redução da massa corporal. Porém, o impacto do exercício contra-resistência, incluindo o consumo de oxigênio em excesso pós-exercício sobre o gasto energético ainda é questionável. O objetivo desta revisão foi discutir a influência das variáveis do exercício contra-resistência (isto é, intensidade, intervalo de recuperação, velocidade de execução, número de séries, e modo - circuito ou contínuo) sobre o gasto energético durante e após uma sessão de exercícios, envolvendo uma discussão sobre os possíveis mecanismos do consumo de oxigênio em excesso pós-exercício. As inúmeras possibilidades de combinação entre tais variáveis podem favorecer uma variabilidade bastante grande no gasto energético de uma sessão de exercício contra-resistência (aproximadamente de 3 a 10kcal.min-1). Contudo, o volume total de trabalho parece ser determinante no gasto energético da sessão. O consumo de oxigênio em excesso pós-exercício, por outro lado, parece ser afetado pela intensidade. A manipulação das variáveis do exercício contra-resistência pode afetar os processos metabólicos subjacentes ao consumo de oxigênio em excesso pós-exercício, o que inclui ressíntese dos estoques de fosfato de alta energia, ressaturação da oximioglobina e oxihemoglobina, efeitos termogênicos, remoção de lactato, aumento no turnover protéico, e processos desencadeados pela estimulação simpática. Conclui-se que, para indivíduos destreinados e com sobrepeso, a prescrição de escolha poderia ser a de sessões de baixa intensidade e alto volume. Contudo, para indivíduos treinados, o aumento do gasto energético, a partir da execução de sessões de exercício contra-resistência mais intensas, pode ser adequado, dada a influência da intensidade sobre o consumo de oxigênio em excesso pós-exercício. Dessa forma, para a prescrição dietética, o nutricionista deve considerar e se beneficiar dos efeitos do treinamento com exercício contra-resistência sobre o aumento do gasto energético.

Termos de indexação: consumo de oxigênio; emagrecimento; exercício; sobrepeso.

\footnotetext{
1 Laboratório Crossbridges, Programa de Pós-Graduação em Educação Física, Universidade Gama Filho. Rua Manoel Vitorino, 625, Piedade, 20748-900, Rio de Janeiro, RJ, Brasil. Correspondência para/Correspondence to: P.S.C. GOMES. E-mail: crossbridges@ugf.br

2 Departamento de Nutrição, Centro Universitário Metodista Bennett. Rio de Janeiro, RJ, Brasil.

${ }^{3}$ Departamento de Nutrição, Universidade Gama Filho. Rio de Janeiro, RJ, Brasil.
} 


\section{A B S T R A C T}

The increase in energy expenditure through physical activity is recognized as an important component in weight loss programs. The impact of resistance exercise, including excess post-exercise oxygen consumption (correspond to the post-exercise energy expenditure), on energy expenditure, however, remains inconclusive. The purpose of the present review was to discuss the influence of the resistance exercise variables (intensity, rest interval, movement velocity, number of sets, and type - circuit or continuous) on energy expenditure during and after an exercise bout. The excess post-exercise oxygen consumption mechanisms were also discussed. The innumerous possibilities of combinations among resistance exercise variables result in a wide range of energy expenditure values for an exercise session (approximately between 3 to 10kcal.min-1). Nevertheless, volume appears to be determinant in the energy expenditure of resistance exercise itself, excess post-exercise oxygen consumption, on the other side, may be affected by exercise intensity. The manipulation of resistance exercise variables may affect the metabolic processes underlying excess post-exercise oxygen consumption, including resynthesis of high energy phosphates stores, resaturation of oxyhemoglobin and oxymyoglobin, thermogenic effects, lactate removal, increased protein turnover, and effects mediated by sympathetic activity. In conclusion, it might be advisable to use low intensity and high volume exercises in a training session for untrained and overweight subjects. However, trained individuals could benefit from more intense resistance exercise, due to the effects of intensity on excess post-exercise oxygen consumption. Thus, nutritionists should consider the effects of resistance exercise on total energy expenditure in order to prescribe effective diets for weight loss purposes.

Indexing terms: oxygen consumption; weight loss; exercise; overweight.

\section{N T R O D U ÇÃ O}

Há uma crescente preocupação mundial com o aumento da prevalência de sobrepeso e obesidade (índice de massa corporal igual ou superior a $\left.25 \mathrm{~kg} \cdot \mathrm{m}^{-2}\right)^{1}$. A preocupação acontece não somente do ponto de vista estético, visto que compreende o principal motivo pelo qual as pessoas procuram academias de ginástica², mas por o mesmo contribuir, de forma relevante, para a maior incidência de doenças crônicas.

A prática sistemática de atividade física e a restrição energética devem estar presentes em todos os programas destinados ao emagrecimento. As recomendações atuais ${ }^{3}$ incluem a realização, inicialmente, de 30 minutos de atividade física, preferencialmente todos os dias, ou um gasto energético semanal de $1000 \mathrm{kcal}$, progredindo para um gasto energético superior a $2000 \mathrm{kcal}$ semanais. A contribuição do treinamento com exercícios contra-resistência (ECR), também conhecido como musculação, na redução ponderal ainda carece de maiores evidências. O American College of Sports Medicine ${ }^{3}$, no posicionamento sobre emagrecimento, incluiu o ECR entre as recomendações propostas com o objetivo de melhorar a capacidade funcional pelo aumento da força e potência muscular, além de aumentar o gasto energético diário. Ainda, ao falar de dispêndio energético da atividade física, deve-se considerar o custo envolvido no retorno à homeostase, que pode ser observado pelo consumo de oxigênio em excesso pós-exercício (EPOC - excess post-exercise oxygen consumption).

O conhecimento do gasto energético do exercício e das respostas metabólicas por ele desencadeadas pode ser importante para auxiliar no cálculo das necessidades energéticas diárias, ou determinar a eficiência do organismo durante a realização de um exercício.

Assim, esta revisão buscou discutir a influência das diversas variáveis do ECR (i.e. intensidade, intervalo de recuperação, velocidade de execução, número de séries, modo - circuito ou contínuo) sobre o gasto energético durante e após uma sessão de ECR, considerando os mecanismos do EPOC e as possíveis implicações sobre o processo de emagrecimento.

\section{Consumo de oxigênio em excesso pós-exercício}

Após a execução de uma sessão de exercícios, seja aeróbio ou contra-resistência, a 
taxa metabólica permanece elevada em relação aos valores de repouso, para que o organismo retorne ao seu estado de equilíbrio. Esse momento, denominado por Gaesser e Brooks ${ }^{4}$ como EPOC, caracteriza-se pelo consumo de oxigênio aumen-tado em relação ao pré-exercício (Figura 1). O consumo de oxigênio guarda relação direta com o gasto energético, ou seja, considerase que a cada litro de $\mathrm{O}_{2}$ consumido, aproximadamente $5 \mathrm{kcal}$ são geradas no organismo.

Três componentes do EPOC foram identificados: o componente rápido, com duração de 10 segundos a alguns minutos; o lento, que pode durar várias horas, dependendo do grau de distúrbio à homeostase causado pela atividade ${ }^{5}$; e o ultralento, observado pela taxa metabólica em valores elevados por até $48 \mathrm{~h}$ após o exercício ${ }^{6}$. Os maiores valores de EPOC são observados nos minutos iniciais da fase de recuperação. Durante o componente lento, os valores são muito menos pronunciados, embora nessa fase ainda existam processos visando a restauração da homeostase que, no entanto, requer uma menor captação de oxigênio do que os processos que ocorrem na fase rápida?.

O EPOC parece depender da extensão dos distúrbios metabólicos causados pelo exercício. É importante ressaltar que os estudos sobre os

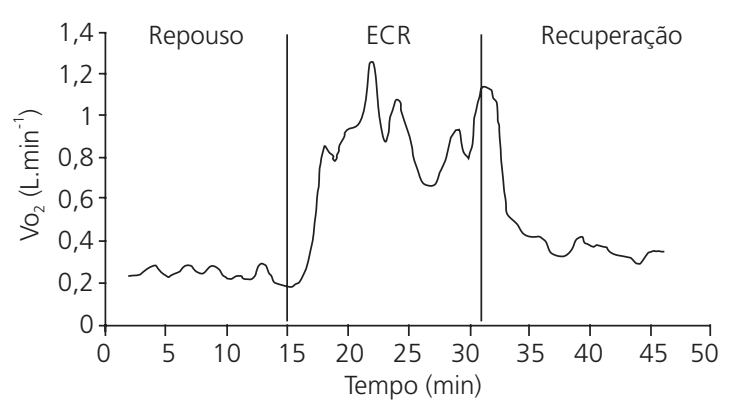

Figura 1. Exemplo típico do consumo de oxigênio antes, durante e após uma sessão de exercícios contra-resistência (ECR; 1 série de 8 repetições a 70\% de 1 repetição máxima para 7 exercícios realizados por um sujeito do sexo masculino; dados não publicados do nosso laboratório, Rio de Janeiro, 2005). possíveis mecanismos do EPOC foram realizados em animais ou em humanos em atividade aeróbia. Muito pouco se sabe sobre os mecanismos do EPOC após o exercício contra-resistência.

\section{Mecanismos responsáveis pelo componente rápido}

\section{Restauração na concentração de fosfatos de alta energia}

O exercício contra-resistência leva a uma depleção parcial nos estoques de adenosina trifosfato (ATP) e quase total de creatina fosfato $(C P)^{8}$, sendo a magnitude da depleção dependente da intensidade da contração muscular ${ }^{9}$.

A refosforilação dos estoques de ambos os substratos energéticos é um processo que depende do fornecimento de oxigênio ${ }^{10}$, sendo o ATP utilizado para refosforilação da creatina derivado do metabolismo aeróbio ${ }^{9}$. Pela restauração, completa em poucos minutos, sua principal contribuição ocorre no componente rápido do EPOC ${ }^{11}$. Após um exercício máximo, esse processo pode ser responsável por até $10 \%$ do volume total de EPOC ${ }^{12}$.

\section{Ressaturação da oxihemoglobina e oximioglobina}

Há uma redução na quantidade de oxigênio no sangue venoso misto com o exercício, que parece apresentar uma relação linear com o aumento da intensidade, ao menos na atividade aeróbia $^{11}$. De forma similar, parte do oxigênio ligado à mioglobina também é utilizado, principalmente no início do exercício ${ }^{13}$.

Segundo Bahr ${ }^{11}$, a contribuição total da ressaturação da oxihemoglobina e da oximioglobina é de cerca de $0,3 \mathrm{~L} \mathrm{O}_{2}$, sendo que tal processo acontece em poucos minutos. Sua contribuição para o EPOC, portanto, acontece somente no componente rápido. 


\section{Mecanismos responsáveis pela fase lenta}

\section{Efeitos termogênicos}

Segundo Gaesser \& Brooks ${ }^{4}$, o principal fator contribuinte para o aumento na taxa metabólica pós-exercício é a temperatura corporal elevada. Há um aumento na atividade enzimática com a elevação na temperatura corporal, sendo possível esperar uma relação direta entre o consumo de oxigênio e a temperatura. Em temperaturas fisiológicas elevadas, verificou-se uma redução na eficiência fosforilativa (razão adenosina difosfato (ADP):oxigênio) e uma maior taxa respiratória mitocondrial na ausência de ADP exógeno ${ }^{12}$, atribuídos a um aumento na atividade da ATPase mitocondrial.

O exercício contra-resistência induz aumentos na temperatura central e muscular. Após a realização de extensão isotônica de joelho por 15min, foi observada uma elevação na temperatura esofageana e muscular (vasto lateral) de $0,55^{\circ} \mathrm{C}$ e $3,2^{\circ} \mathrm{C}$, respectivamente, que persistiu por até 60 minutos $^{14}$. No período pós-exercício, o retorno da temperatura muscular aos valores pré-exercício apresenta um comportamento bastante similar ao $\mathrm{EPOC}^{15}$. A temperatura elevada é responsável por $60 \%$ a $70 \%$ da magnitude da fase lenta do EPOC após sessões de exercício aeróbio com intensidades entre $50 \%$ e $80 \%$ do $\mathrm{VO}_{2}$ máx ${ }^{16}$.

\section{Estimulação simpática}

O aumento na atividade simpato-adrenal pode constituir um dos mecanismos responsáveis pelo EPOC, em especial pelo componente prolongado ${ }^{5}$, visto que a epinefrina e a norepinefrina, por meio de receptores $\beta$, são potentes estimuladores do metabolismo energético. Durante o exercício físico, ao menos do tipo aeróbio, há um aumento na concentração plasmática de catecolaminas, que ocorre de forma linear com a duração e exponencial com a intensidade.
O retorno das concentrações plasmáticas de catecolaminas aos níveis de repouso acontece de forma mais rápida que o retorno da captação de oxigênio, mas é importante ressaltar que a concentração plasmática de catecolaminas pode ser um indicador insensível da atividade simpática, visto que a taxa de clearance é bastante elevada, além da maior sensibilidade às catecolaminas após o exercício ${ }^{5}$.

As catecolaminas podem exercer seus efeitos termogênicos indiretamente. Tem sido observado um aumento na oxidação de ácidos graxos e no ciclo triacilglicerol-ácidos graxos (ciclo fútil) após o exercício, sendo as catecolaminas reguladoras essenciais desse processo. No ciclo fútil, os ácidos graxos liberados durante a lipólise são reesterificados a triacilgliceróis ao invés de serem oxidados, num processo dependente de ATP. O custo energético associado ao aumento desse ciclo pode ser responsável por uma parte significativa do EPOC após o exercício ${ }^{17}$.

No entanto, estudos realizados com a administração de $\beta$-bloqueadores seletivos (atenolol) e não seletivos (propanolol) não suportam um papel da atividade simpática sobre a taxa metabólica após o exercício ${ }^{18}$, o que sugere uma regulação dos efeitos simpáticos pelos receptores $\beta$ sobre a taxa metabólica de repouso, mas não após o exercício.

A principal contribuição da atividade simpática sobre O EPOC parece estar nos processos metabólicos e bioquímicos estimulados durante o exercício, que são lentamente revertidos após o término do mesmo, inclusive na ausência de uma maior atividade simpática nesse período. Durante o exercício, as catecolaminas estimulam a glicogenólise, glicólise e lipólise, influenciam a liberação de outros hormônios, e modulam o tônus vascular. Tais aspectos podem ser importantes para a regulação do fluxo sangüíneo e do consumo de oxigênio pelos tecidos após o exercício. 


\section{Remoção de lactato}

O lactato foi, durante muito tempo, conhecido como o "vilão do metabolismo", considerado o produto final (sem utilidade) da glicólise em condições de hipóxia, a principal causa do débito de oxigênio, o principal causador da fadiga muscular, e um fator chave na lesão tecidual induzida pela acidose ${ }^{19}$. Hoje, sabe-se que o lactato desempenha um papel fundamental no metabolismo. A remoção do lactato ocorre via oxidação a moléculas de dióxido de carbono e água (cerca de 75\%) e gliconeogênese (cerca de $25 \%)$.

O exercício contra-resistência induz um aumento significativo na concentração plasmática de lactato ${ }^{8}$, dependente da intensidade ${ }^{7}$. A sua velocidade de remoção apresenta uma relação linear direta $(r=0,86 ; p<0,05)$ com o EPOC durante a primeira hora após exercício ${ }^{11}$, sugerindo uma contribuição para o componente lento do EPOC.

\section{Alteração no substrato energético}

Após o ECR, há uma alteração no substrato energético predominante. Tesch et al. ${ }^{8}$ observaram uma contribuição substancial dos fosfatos de alta energia e da glicólise e glicogenólise para o fornecimento de energia durante o exercício. Já durante O EPOC, Os lipídeos parecem ser o principal substrato energético ${ }^{20,21}$. Jamurtas et al. ${ }^{20}$ mediram o quociente respiratório e verificaram que o mesmo permaneceu abaixo dos valores de repouso por até 24 h após uma sessão de $E C R$, duração esta, igual à do EPOC. Orsmbee et al. ${ }^{21}$ também observaram aumento na oxidação de lipídeos por meio de microdiálise após o ECR. Em contrapartida, Melanson et al..$^{22}$, utilizando calorimetria indireta, não observaram aumento da lipólise pós-ECR comparada ao controle. Segundo Bahr ${ }^{11}$, tal modificação no predomínio do substrato oxidado pode contribuir com cerca de $10 \%$ a $15 \%$ do EPOC.

\section{Componente ultralento}

Os estudos que demonstraram o componente ultralento apresentaram algumas características em comum. Jamurtas et al. ${ }^{20}$, Melby et al..$^{23}$, e Schuenke et al. ${ }^{24}$ adotaram um protocolo similar, incluindo pelo menos quatro séries a 70\%-75\% de uma repetição máxima (RM). Em cada série, os indivíduos realizavam o maior número de repetições possível, até a fadiga. O protocolo realizado foi bastante intenso, com os sujeitos relatando náuseas e desconforto.

No estudo de Dolezal et al. ${ }^{6}$ os indivíduos realizaram oito séries de 6 RM no leg press, com $4 \mathrm{~s}$ de duração da fase excêntrica. A taxa metabólica apresentou-se elevada por até 48h após o término da sessão. Sabe-se que o treinamento excêntrico induz uma maior lesão muscular ${ }^{25}$, sendo reportadas taxas de turnover protéico aumentadas por até dois dias, em resposta aguda a uma série intensa de ECR excêntricos. Parte do aumento na taxa metabólica pode ser creditada ao custo energético do turnover protéico, visto que o mesmo pode corresponder a até $20 \%$ do metabolismo de repouso ${ }^{26}$.

Um outro aspecto que pode influenciar a resposta metabólica ao ECR diz respeito ao nível de treinamento dos indivíduos. Apesar de a taxa metabólica apresentar-se elevada em relação aos valores da linha de base em ambos os grupos (treinados e destreinados) envolvidos no estudo de Dolezal et al. ${ }^{6}$, os treinados apresentaram uma resposta significativamente atenuada, quando comparados aos destreinados ( 5\%).

O componente ultralento parece ocorrer apenas após sessões nas quais um extenso distúrbio à homeostase foi acarretado pelo exercício físico, uma vez que esteve presente após sessões intensas e com um componente excêntrico.

\section{Dispêndio energético e EPOC em uma sessão de ECR}

No treinamento físico, o delineamento apropriado do programa de exercícios é um 
aspecto fundamental para que os objetivos sejam atingidos, o que se estende a programas destinados à redução ponderal. Nesse caso, um ponto importante a ser observado é o gasto energético da mesma, além do EPOC associado. Durante uma sessão de $E C R$, o gasto energético pode ser de pequena magnitude (50 a $115 \mathrm{kcal})^{7,27,28}$, embora valores de até $864 \mathrm{kcal}$ em uma única sessão, excluindo o EPOC, tenham sido reportados $^{24}$. Com relação ao EPOC, valores de $6 \mathrm{kcal}$ a $114 \mathrm{kca} \mid 6,7,24,29$, e duração de $14 \mathrm{~min}$ a $48 \mathrm{~h}$ têm sido reportados após uma sessão de ECR (Anexo). Tal diversidade de resultados parece ser decorrente das inúmeras possibilidades de combinação entre as variáveis do ECR. O impacto de cada uma delas será discutido adiante, embora deva ser considerada a interdependência entre as mesmas. Ainda, características individuais da amostra, tais como idade, sexo, nível de treinamento e quantidade de massa livre de gordura (MLG), devem ser consideradas ${ }^{30}$.

Cabe ressaltar que geralmente se utiliza a medida de consumo de $\mathrm{O}_{2}$ para estimativa do gasto energético. Entretanto, a participação do metabolismo anaeróbio poderia representar até $39 \%$ do custo energético no ECR ${ }^{31}$. Assim, os resultados de estudos que utilizaram este método devem ser interpretados com cautela, pois os valores reportados podem ter sido subestimados.

Pelo fato de a MLG ser um importante determinante da taxa metabólica ${ }^{32}$, o gasto energético em homens é sempre significativamente mais alto, em valores absolutos, do que em mulheres, considerando protocolos similares de exercício. Quando expresso de forma relativa, por kg de MLG, o gasto é similar, exceto no estudo de Morgan et al. ${ }^{33}$ no qual, para a mesma tarefa, as mulheres apresentaram um maior gasto de energia que os homens.

Após revisão extensa da literatura, Meirelles \& Gomes $^{30}$ concluíram que, quando analisado individualmente, o volume parece ser a variável de maior impacto sobre o gasto ener- gético durante o exercício. Contudo, somente em sua definição, o volume (produto do número de séries, repetições e carga) envolve três variáveis do $E C R$, evidenciada a inter-relação entre as mesmas. Faz-se necessário, portanto, controlar todas as variáveis para que se possa conhecer a influência de cada uma delas sobre o dispêndio energético.

\section{Variáveis do treinamento contra-resistência}

\section{Intensidade}

Sessões mais intensas (sendo intensidade definida como percentual de uma determinada carga máxima) de ECR levam a um maior recrutamento de unidades motoras de alto limiar, uso de energia para remoção do lactato, alteração na atividade da miosina ATPase, maior recrutamento dos músculos estabilizadores e respiratórios, e maior atividade simpática ${ }^{34}$. Tais aspectos poderiam reduzir a eficiência do trabalho para o mesmo volume, aumentando o dispêndio energético para realizar a atividade. Entretanto, a intensidade parece não apresentar uma relação direta com o gasto energético durante a sessão de $\mathrm{ECR}^{30}$. Quando realizadas com o mesmo volume, sessões de intensidades distintas ocasionaram custo energético de igual magnitude 7 . Ao considerar protocolos com volumes diferentes, a intensidade também não influenciou o dispêndio energético $^{30,35}$.

Por outro lado, o EPOC parece ser influenciado pela intensidade ${ }^{7}$, principalmente no componente rápido. Tal fato pode ser explicado por a mesma determinar a taxa de depleção dos estoques de CP, glicogênio muscular e ATP, e a restauração dessas reservas energéticas ocorrer durante o componente rápido. Thornton \& Potteiger ${ }^{7}$ observaram uma maior concentração de lactato plasmático no grupo que realizou o ECR mais intenso. O EPOC, medido por $2 \mathrm{~h}$, foi significativamente mais elevado (2,2 versus $1,1 \mathrm{~L}$ $\mathrm{O}_{2}$ ) após a sessão mais intensa ( $85 \%$ de $8 \mathrm{RM}$ ) do que a menos intensa (45\% 8 RM). 
Contrariando tais achados, Olds \& Abernethy ${ }^{36}$ não verificaram influência da intensidade sobre O EPOC, apesar de uma tendência para um maior consumo de oxigênio em todas as medidas realizadas no grupo que treinou mais intensamente. Alguns aspectos do estudo devem, entretanto, ser ressaltados. A diferença entre as intensidades foi pequena ( $75 \%$ e $60 \%$ 1RM), podendo não ter sido suficiente para provocar um efeito do tratamento. $O$ estudo apresentou outras limitações, como ampla faixa etária (22 a 55 anos), grandes diferenças interindividuais na duração (1 a $5 \mathrm{~h}$ ) e magnitude ( 27 a $135 \mathrm{kcal}$ ) do EPOC. Os primeiros minutos após o exercício, quando o EPOC é mais elevado, não foram medidos.

Além da magnitude, a duração do EPOC também parece sofrer influência da intensidade. Como reportado anteriormente, somente após sessões intensas observou-se o componente ultralento do EPOC. Schuenke et al. ${ }^{24}$ observaram que o consumo de oxigênio permaneceu elevado por até 38h, em relação aos valores de repouso, após a realização de quatro séries de 10 RM para três exercícios. Nas 48 h após a sessão de $E C R$, o consumo de oxigênio foi, em média, $0,66 \mathrm{ml}$ $\mathrm{O}_{2} \cdot \mathrm{kg}^{-1} \cdot \mathrm{min}^{-1}$ superior ao repouso, equivalendo a um aumento de cerca de $20 \%$ no metabolismo. Para um homem de $70 \mathrm{~kg}$, representaria um adicional de $330 \mathrm{kcal}$ por dia, o que sugere que a energia necessária para recuperação de uma sessão de ECR pode ter uma contribuição positiva significativa em programas de controle do peso. Por outro lado, nos estudos em que o EPOC não teve longa duração, a intensidade foi muito baixa $(75 \% 20 \mathrm{RM})^{37}$ ou o número de repetições foi muito pequeno $(3 \text { a } 6)^{38}$.

Sendo assim, a intensidade parece ser determinante da magnitude e duração do EPOC, mas não do gasto energético da sessão de ECR propriamente dita.

\section{Intervalo de recuperação entre séries}

A manipulação do intervalo de recuperação pode ser vista como um aumento na intensidade, pois um período mais curto de recuperação pode aumentar o estresse induzido pelo exercício de forma similar a um aumento na carga ${ }^{7}$.

Haltom et al..$^{37}$ testaram dois intervalos diferentes (20s vs 60s) e verificaram um dispêndio energético relativo (por unidade de tempo) na situação intervalo curto $(8,5 \mathrm{kcal} \cdot \mathrm{min}-1)$ significativamente superior à de intervalo longo $(6,7 \mathrm{kcal} \cdot \mathrm{min}-1)$. O EPOC, medido por uma hora após o término da sessão, foi significativamente mais alto na situação intervalo curto em relação ao longo (52 vs $37 \mathrm{kcal})$. Tais resultados foram corroborados por De Groot et al. ${ }^{35}$, que observaram um gasto de energia de uma sessão de ECR com intervalo curto (30s) aproximadamente 20\% superior ao longo (60s).

A adoção de intervalos mais curtos de recuperação entre séries parece levar a um maior custo energético da sessão, quando expresso por tempo de trabalho, e a um EPOC de maior magnitude.

\section{Velocidade de execução}

A velocidade com que cada repetição é realizada parece afetar as respostas neurais, hipertróficas e metabólicas ao ECR, embora seja uma variável pouco estudada, principalmente nos exercícios dinâmicos não isocinéticos. Hunter et al. ${ }^{28}$ compararam o efeito do exercício executado de acordo com o método super lento (concêntrica 10s, excêntrica 5s) e velocidade livre, ambos com 29min de duração. Os autores demonstraram um maior gasto energético durante (114 vs 73,5kcal) e após (41 vs 33,5kcal em 15 minutos) a sessão em velocidade livre. Pode ser que o volume total de treino tenha interferido na resposta, pois o grupo de velocidade livre realizou duas séries de cada exercício, contra uma do super lento. Já Ballor et al.$^{39}$ não observaram um efeito da velocidade sobre o dispêndio energético da sessão. No entanto, além dos diferentes protocolos de treinamento, nesse último estudo foram adotados equipamentos hidráulicos, o que dificulta a comparação entre os resultados obtidos. 
Pelos dois estudos até então realizados, não é possível estabelecer o impacto da velocidade de execução do movimento sobre o gasto energético e EPOC.

\section{Número de séries}

É recomendada, por algumas instituições na área de saúde ${ }^{40,41}$, a inclusão de pelo menos uma série de 8 a 15 repetições para os principais grupamentos musculares em programas destinados à melhoria da saúde e qualidade de vida. Tal recomendação baseia-se em estudos que demonstraram que programas de série única podem levar a ganhos em força e hipertrofia muscular de forma similar a séries múltiplas ${ }^{42}$, principalmente em indivíduos sedentários ou na fase inicial de um programa de treinamento ${ }^{43}$.

A elaboração de um programa de ECR deve considerar, essencialmente, o nível de treinamento, os objetivos e o tempo disponível do indivíduo. Dessa forma, identificar se há uma relação tempo-benefício que justifique a realização de três vezes mais trabalho pode ser importante em qualquer tipo de programa, inclusive nos destinados ao emagrecimento.

Phillips \& Ziuraitis ${ }^{44}$ observaram um gasto energético de $135 \mathrm{kcal}$ para homens e $82 \mathrm{kcal}$ para mulheres, em uma sessão de série única. Os mesmos autores realizaram um estudo similar em idosos e observaram um gasto energético $40 \%$ inferior (84kcal) em homens e 15\% inferior (70kcal) em mulheres, comparado ao obtido em jovens ${ }^{45}$. Tal diferença foi creditada à menor massa corporal dos idosos e à menor carga levantada em cada um dos oito exercícios que compunham a sessão de ECR.

Henley et al. ${ }^{46}$ e Haddock \& Wilkins ${ }^{47}$ demonstraram que o gasto energético total de três séries foi, aproximadamente, três vezes superior ao encontrado com séries simples. Entretanto, quando os valores foram expressos em relação ao tempo de exercício, o gasto foi semelhante. 0
EPOC foi de igual magnitude em valores absolutos e maior na situação série única, quando expresso por tempo de exercício.

Apesar da escassez de estudos comparando o gasto energético de séries simples e múltiplas, acredita-se que o gasto energético absoluto da sessão aumente proporcionalmente com o número de séries realizadas, provavelmente pelo maior volume realizado, enquanto parece não haver diferença no gasto relativo ao tempo de duração do estímulo. A magnitude do EPOC, por outro lado, parece ser menos afetada pelo número de séries executadas.

\section{Sessões de ECR no modo circuito ou contínuo}

O ECR pode ser realizado de maneira contínua, na qual um determinado número de séries de um mesmo exercício é executado antes que se execute outro exercício; ou em circuito, no qual uma série de cada exercício é realizada antes de uma nova série.

Elliot et al. ${ }^{38}$ e Pinchon et al. ${ }^{48}$ compararam os efeitos sobre o gasto energético entre sessões de ECR, realizadas em circuito e de forma contínua, e reportaram um maior gasto no modo circuito durante a sessão $(9,1$ vs $6,2 \mathrm{kcal} \cdot \mathrm{min}-1$ e 4,9 vs $4,5 \mathrm{kcal} \cdot \mathrm{min}-1$ para circuito e contínuo, respectivamente $\left.{ }^{38,48}\right)$. Não houve diferenças significativas no EPOC (cerca de $10 \mathrm{~L} \mathrm{O}_{2}$ ). Alguns aspectos de ambos os estudos, contudo, devem ser ressaltados. Os estudos não diferiram apenas no modo de execução; os indivíduos foram submetidos a um maior volume de trabalho na situação circuito, sendo os números de séries e repetições, a intensidade e os intervalos de recuperação diferentes entre as condições testadas, o que prejudica qualquer comparação e posterior conclusão.

A realização de sessões de ECR com baixa intensidade e alto número de repetições pode acarretar um gasto de energia similar a altas intensidades e reduzido número de repetições. Tal 
informação é particularmente relevante ao considerar indivíduos sedentários e com sobrepeso, cuja preocupação seja a redução ou o controle da massa corporal, já que a aderência à atividade é ponto fundamental. Embora ainda não existam relatos que associem a intensidade do ECR à aderência ao mesmo, programas de atividade aeróbia com intensidades altas estão associados a maiores taxas de abandono ${ }^{49}$.

A contribuição do EPOC para o aumento no dispêndio energético diário parece ser importante somente após sessões muito intensas. Essa informação pode ser importante na prescrição do treinamento com ECR para indivíduos já treinados, que buscam reduzir a gordura corporal. Para indivíduos iniciantes e com sobrepeso, para os quais se indicam sessões de menor intensidade, 0 gasto energético adicional após o ECR é baixo, provavelmente não tendo uma influência significativa sobre o dispêndio energético total e a subseqüente perda de peso. Conclui-se que, para indivíduos destreinados e com sobrepeso, a prescrição de escolha poderia ser a de sessões de baixa intensidade e alto volume. Dessa forma, para indivíduos treinados, o aumento do gasto ener-gético a partir da execução de sessões de ECR mais intensas pode ser adequado, dada a influência da intensidade sobre o EPOC.

\section{O N CLUS Ã O}

Os diversos resultados disponíveis acerca do gasto energético do ECR ainda não são suficientes para determinar o efeito isolado de cada uma das suas variáveis. Tal dificuldade decorre das inúmeras possibilidades de combinação entre as variáveis do ECR, além da interferência de características individuais, como sexo, nível de aptidão física e, principalmente, a quantidade de massa livre de gordura e de gordura corporal. No entanto, é conhecido que o volume total de trabalho é a variável de maior impacto no gasto energético durante a execução da atividade. Já o consumo de oxigênio em excesso pós-exercício, parece ser fortemente afetado pela intensidade, pois sessões de exercícios mais intensas acarretam um maior distúrbio à homeostase. Do ponto de vista nutricional, o aumento do gasto energético com a atividade física pode facilitar a geração de um balanço energético negativo, evitando que a dieta tenha que ser extremamente restritiva, uma vez que dietas de muito baixo valor energético são, geralmente, inadequadas em micronutrientes.

Sugere-se a realização de estudos adicionais que estimem o gasto energético total de sessões de ECR cujas variáveis sejam bem controladas, ou seja, os resultados deveriam expressar a soma do dispêndio energético durante a atividade e o EPOC. Dessa forma, a prescrição de dietas para redução ponderal poderia levar em consideração estimativas mais aproximadas do gasto energético diário do indivíduo alvo da consulta. Além disso, seria de grande valia a investigação dos mecanismos associados ao EPOC no ECR, uma vez que os mecanismos hoje descritos na literatura advêm de pesquisas com atividades aeróbias.

\section{REFERÊ N CIAS}

1. World Health Organization. Obesity: Preventing and managing the global epidemic. Geneva; 1998. Report of a WHO Consultation on Obesity.

2. Gomes PSC, Santos TM. Por que as pessoas procuram uma academia? Rev ACAD. 2004; 21: 8-9.

3. American College of Sports Medicine. Position stand on the appropriate intervention strategies for weight loss and prevention of weight regain for adults. Med Sci Sports Exerc. 2001; 33(12): 2145-56.

4. Gaesser GA, Brooks GA. Metabolic basis of excess post-exercise oxygen consumption: a review. Med Sci Sports Exerc. 1984; 16(1):29-43.

5. Børsheim E, Knardahl S, Hostmark AT, Bahr R. Adrenergic control of post-exercise metabolism. Acta Physiol Scand. 1998; 162(3):313-23.

6. Dolezal BA, Potteiger JA, Jacobse DJ, Benedict SH. Muscle damage and resting metabolic rate after acute resistance exercise with an eccentric overload. Med Sci Sports Exerc. 2000; 32(7):1202-7.

7. Thornton MK, Potteiger JA. Effects of resistance exercise bouts of different intensities but equal 
work on EPOC. Med Sci Sports Exerc. 2002; 34(4):715-22.

8. Tesch PA, Colliander EB, Kaiser P. Muscle metabolism during intense, heavy resistance exercise. Eur J Appl Physiol Occup Physiol. 1986; 55(4):362-6.

9. McMahon S, Jenkins D. Factors affecting the rate of phosphocreatine resynthesis following intense exercise. Sports Med. 2002; 32(12):761-84.

10. Idstrom JP, Subramanian V, Chance B, Schersten T, Bylund-Fellenius A. Oxygen dependence of energy metabolism in contracting and recovering rat skeletal muscle. Am J Physiol. 1985; 248(1 Pt 2): H40-8.

11. Bahr R. Excess postexercise oxygen consumption: magnitude, mechanisms and practical implications. Acta Physiol Scand Suppl. 1992; 605:1-70.

12. Brooks GA, Hittelman KJ, Faulkner JA, Beyer RE. Temperature, skeletal muscle mitochondrial functions, and oxygen debt. Am J Physiol. 1971; 220(4):1053-9.

13. Clark BJ, Coburn RF. Mean myoglobin oxygen tension during exercise at maximal oxygen uptake. J Appl Physiol. 1975; 39(1):135-44.

14. Kenny GP, Reardon FD, Zaleski W, Reardon ML, Haman F, Ducharme MB. Muscle temperature transients before, during, and after exercise using an intramuscular multisensor probe. J Appl Physiol. 2003; 94(6):2350-7.

15. Brooks GA, Hittelman KJ, Faulkner JA, Beyer RE. Tissue temperatures and whole animal oxygen consumption after exercise. Am J Physiol. 1971; 22(2) $1: 427-31$

16. Hagberg JM, Mullin JP, Nagle FJ. Effect of work intensity and duration on recovery $\mathrm{O}_{2}$. J Appl Physiol. 1980; 48(3):540-44.

17. Børsheim E, Bahr R. Effect of exercise intensity, duration and mode on post-exercise oxygen consumption. Sports Med. 2003; 33(14):1037-60.

18. Børsheim E, Bahr R, Hostmark AT, Knardahl S. Effect of beta-adrenoceptor blockade on post-exercise oxygen consumption and triglyceride/fatty acid cycling. Metabolism. 1998; 47(4):439-48.

19. Gladden LB. Lactate metabolism: a new paradigm for the third millennium. J Physiol. 2004; 558(Pt 1): 5-30.

20. Jamurtas $A$, Koutedalis $Y$, Paschalis $V$, Tofas $T$, Yfanti C, Tsiokanos A, et al. The effects of a single bout of exercise on resting energy expenditure and respiratory exchange ratio. Eur J Appl Physiol. 2004; 92(4-5):393-8

21. Ormsbee MJ, Thyfault JP, Johnson EA, Kraus RM, Choi MD, Hickner RC. Fat metabolism and acute resistance exercise in trained men. J Appl Physiol. 2007. In press.

22. Melanson EL, Sharp TA, Seagle HM, Donahoo WT, Grunwald GK, Peters JC, et al. Resistance and aerobic exercise have similar effects on 24-h nutrient oxidation. Med Sci Sports Exerc. 2002; 34(11):1793-800.

23. Melby C, Scholl C, Edwards G, Bullough R. Effect of acute resistance exercise on post energy expenditure and resting metabolic rate. J Appl Physiol. 1993; 75(4):1847-53.

24. Schuenke MD, Mikat P, McBride JM. Effect of an acute period of resistance exercise on excess post-exercise oxygen consumption: implications for body mass management following a bout of heavy resistance exercise. Eur J Appl Physiol. 2002; 86(5):411-7.

25. Hather B, Tesch P, Buchanan P, Dudley G. Influence of eccentric actions on skeletal muscle adaptations to resistance training. Acta Physiol Scand. 1991; 143(2):177-85.

26. Welle S, Nair KS. Relationship of resting metabolic rate to body composition and protein turnover. Am J Physiol. 1990; 258(6 Pt 1):E990-8.

27. Beckham SG, Earnest CP. Metabolic cost of free weight circuit training. J Sports Med Phys Fitness. 2000; 40(2):118-25.

28. Hunter G, Seelhorst D, Snyder S. Comparison of metabolic and heart rate response to super slow vs. traditional resistance training. J Strength Cond Res. 2003; 17(1):76-81.

29. Hickson JF, Wilmore JH, Buono MJ, Constable SH. Energy cost of weight training exercise. Natl Strength Conditioning Assoc J. 1984; 6:22-3.

30. Meirelles CM, Gomes PSC. Efeitos agudos da atividade contra-resistência sobre o gasto energético: revisitando o impacto das principais variáveis. Rev Bras Med Esp. 2004; 10(2):122-30.

31. Scott CB. Contribution of blood lactate to the energy expenditure of weight training. J Strength Cond Res. 2006; 20(2):404-11

32. Zurlo F, Larson K, Bogardus C, Ravussin E. Skeletal muscle metabolism is a major determinant of resting energy expenditure. J Clin Invest. 1990; 86(5):1423-7.

33. Morgan B, Woodruff SJ, Tiidus PM. Aerobic energy expenditure during recreational weight training in females and males. J Sports Sci Med. 2003; $2: 117-22$

34. Hunter GR, Weinsier RL, Bamman MM, Larson DE. A role for high intensity exercise on energy balance and weight control. Int J Obes Metab Disord. 1998; 22(6):489-93. 
35. De Groot DW, Wuinn TI, Kertzer R, Vroman NB, Olney WB. Circuit weight training in cardiac patients: determining optimal workloads for safety and energy expenditure. J Cardiopulm Rehab. 1998; 18(2):145-52.

36. Olds TS, Abernethy PJ. Postexercise oxygen consumption following heavy and light resistance exercise. J Strength Cond Res. 1993; 7(3):147-52.

37. Haltom RW, Kraemer RR, Sloan RA, Hebert EP, Frank $\mathrm{K}$, Tryniecki JL. Circuit weight training and its effects on excess postexercise oxygen consumption. Med Sci Sports Exerc. 1999; 31(11):1613-8.

38. Elliot DL, Goldberg L, Kuelh K. Effect of resistance training on excess post exercise oxygen consumption. J Appl Sport Sci Res. 1992; 6(2): 77-81.

39. Ballor DL, Becque D, Katch VL. Energy output during hydraulic resistance circuit exercise for male and females. J Appl Sport Sci Res. 1989; 3(1):7-12.

40. American College of Sports Medicine. The recommended quantity and quality of exercise for developing and maintaining cardiorespiratory and muscular fitness, and flexibility in healthy adults. Med Sci Sports Exerc. 1998; 30(6):975-91.

41. American Heart Association. Exercise standards for healthcare professionals from the American Heart Association. Circulation. 2001; 104(14):1694-740.

42. Starkey DB, Pollock ML, Ishida Y, Welsch MA, Brechue WF, Graves JE, et al. Effect of resistance training volume on strength and muscle thickness. Med Sci Sports Exerc. 1996; 28(10):1311-20.
43. Wolfe BL, Lemura LM, Cole PJ. Quantitative analysis of single vs. multiple set programs in resistance training. J Strength Cond Res. 2004; 18(1):35-47.

44. Phillips WT, Ziuraitis JR. Energy cost of the ACSM single-set resistance training protocol. J Strength Cond Res. 2003; 17(2):350-5.

45. Phillips WT, Ziuraitis JR. Energy cost of single-set resistance training in older adults. J Strength Cond Res. 2004; 18(3):606-9.

46. Henley MO, Irving BA, Gaesser GA. Effect of single and multiple-set resistance exercise on postexercise energy expenditure [abstract]. Med Sci Sports Exerc. 2004; 36(5):S277.

47. Haddock BL, Wilkins LD. Resistance training volume and postexercise energy expenditure. Int J Sports Med. 2006; 27: 143-8.

48. Pinchon CE, Hunter GR, Morris M, Bond RL, Metz J. Blood pressure and heart rate response and metabolic cost of circuit versus traditional weight training. J Strength Cond Res. 1996; 10(3):153-6.

49. Lee JY, Jensen BE, Oberman A, Fletcher $G$, Fletcher $\mathrm{G}$, Raczynski J. Adherence in the Training Levels Comparison trial. Med Sci Sports Exerc. 1996; 28(1): 47-52.
Recebido em: 26/7/2005

Versão final reapresentada em: 20/6/2006

Aprovada em: 3/8/2006 
ANEXO

RESUMO DOS EFEITOS AGUDOS DE UMA SESSÃO DE EXERCÍCIO CONTRA-RESISTÊNCIA SOBRE O GASTO ENERGÉTICO E O CONSUMO DE OXIGÊNIO EM EXCESSO PÓS-EXERCÍCIO SEGUNDO VÁRIOS AUTORES

\begin{tabular}{|c|c|c|c|c|c|c|}
\hline Autor & Amostra (idade) & Protocolo ECR & & $\mathrm{GE}\left(\mathrm{kcal} \cdot \mathrm{min}^{-1}\right.$ (total)) & EPOC (kcal) & Medida \\
\hline Hickson et al. ${ }^{29}$ & 4 HT (19-26) & $\begin{array}{l}\text { Ex: 5, DS: } 36, \text { I:60s } \\
3 \times 30 \text { s, } 75-80 \% 1 \mathrm{RM}\end{array}$ & 7,4 & dp 1,6 (267 dp 58) & $22 \mathrm{dp} 11$ & $0-14 \min$ \\
\hline Ballor et al. ${ }^{39}$ & $\begin{array}{l}35(2 \mathrm{OH}, 15 \mathrm{M}) \\
\mathrm{T}(\sim 24)\end{array}$ & $\begin{array}{l}\text { C, Ex: } 9 \text {, DS: } 27, \text { I: } 30 \text { s } \\
\text { 3x30s, Hidráulico } \\
\text { Vel: rápida }(R) / \text { média }(M) / \text { lenta } \\
\text { (L) }\end{array}$ & $\begin{array}{l}\text { R) } \\
\text { M) } \\
\text { L) }\end{array}$ & 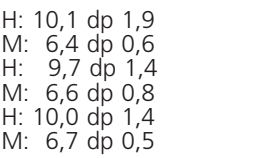 & --- & -- \\
\hline Elliot et al. ${ }^{38}$ & $\begin{array}{l}9(4 \mathrm{H}, 5 \mathrm{M}) \\
\mathrm{T}(\sim 25,5)\end{array}$ & $\begin{array}{l}\text { Ex: } 8 \text {, DS: } 40 \\
\text { C) } 4 \times 15 \mathrm{r}, 50 \% \text { 1RM, I: } 30 \mathrm{~s} \\
\text { Continuo (CO): } 3 \times 3-8 \mathrm{RM}, \\
80-90 \% 1 \mathrm{RM}, 1: 60-120 \mathrm{~s}\end{array}$ & $\begin{array}{l}\text { C) } \\
\text { T) }\end{array}$ & $\begin{array}{l}9,0(362 \mathrm{dp} \mathrm{167)} \\
6,2(248 \mathrm{dp} \mathrm{129)*}\end{array}$ & $\begin{array}{l}\text { C) } 48 \mathrm{dp} 20 \\
\text { T) } 51 \mathrm{dp} 31^{*}\end{array}$ & 0-90min \\
\hline Melby et al. ${ }^{23}$ & 7 HT (20-40) & $\begin{array}{l}\text { Ex: } 10,8-12 \mathrm{RM}, 70 \% 1 \mathrm{RM} \\
\text { 1) } 6 \text { séries, I: } 3 \mathrm{~min} \\
\text { 2) } 5 \text { séries, I: } 4 \mathrm{~min}\end{array}$ & $\begin{array}{l}\text { 2) } 7 \\
\text { med }\end{array}$ & $\begin{array}{l}\text { 7e } 9 \text { (661 e 864) } \\
\text { dido em } 2 \text { indivíduos }\end{array}$ & $\begin{array}{l}\text { 1) } 35 \mathrm{dp} 1 \text {; TMR 9,4\%> } \\
\text { 2) } 36 \mathrm{dp} 6 \text {; TMR 4,7\%> }\end{array}$ & $\begin{array}{l}\text { 0-120min } \\
\text { TMR 15h }\end{array}$ \\
\hline Olds \& Abernathy ${ }^{36}$ & 7 HT (20-55) & $\begin{array}{l}\text { C, Ex: 7, I: 3,5min } \\
\text { IB) } 2 \times 15 r, 60 \% 1 \mathrm{RM} \\
\text { IA) } 2 \times 12 \mathrm{r}, 75 \% 1 \mathrm{RM}\end{array}$ & & -- & $\begin{array}{l}\text { IB) } 31 \mathrm{dp} 33 \\
\text { IA) } 39 \mathrm{dp} 40\end{array}$ & $0-60 \mathrm{~min}$ \\
\hline Pinchon et al. ${ }^{48}$ & $\begin{array}{l}8(5 \mathrm{H}, 3 \mathrm{M}) \\
(23-34)\end{array}$ & $\begin{array}{l}\text { Ex: } 4 \\
\text { C) } 2 \times 20 r, 47 \% \text { 1RM, I: } 30 \mathrm{~s} \\
\text { Continuo (CO): } 2 \times 10 \text { r, } 69 \% \\
\text { 1RM, I: } 90 \text { s }\end{array}$ & $\begin{array}{l}\text { C) } 2 \\
\text { CO) } 2\end{array}$ & $\begin{array}{l}\text { 4,9 dp 1,9 (53 dp 21) } \\
4,5 \mathrm{dp} 2,0(50 \mathrm{dp} 23)\end{array}$ & --- & ---- \\
\hline De Groot et al. ${ }^{35}$ & $\begin{array}{l}\text { 9H DT C/ DAC } \\
(54-75)\end{array}$ & $\begin{array}{l}\text { C, Ex: 6, 3x30s } \\
\text { 1) } 60 \% 1 \mathrm{RM} \text {; I: } 30 \mathrm{~s} \\
\text { 2) } 60 \% 1 \mathrm{RM} \text {; I: } 60 \mathrm{~s} \\
\text { 3) } 40 \% 1 \mathrm{RM} ; \mathrm{I}: 30 \mathrm{~s} \\
\text { 4) } 40 \% 1 \mathrm{RM} ; \mathrm{I}: 60 \mathrm{~s}\end{array}$ & $\begin{array}{l}\text { 1) } \\
\text { 2) } \\
\text { 3) } \\
\text { 4) } \\
3\end{array}$ & 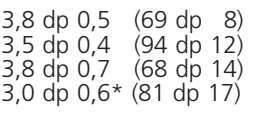 & -- & - \\
\hline Haltom et al. ${ }^{37}$ & 7 HT (27 dp 1) & $\begin{array}{l}\text { C, Ex: } 8 \\
\text { 2x20r, } 75 \% \text { 20RM } \\
\text { I curto (IC): 20s, DS: } 13 \\
\text { I longo (IL): 60s, DS: } 23\end{array}$ & $\begin{array}{l}\text { IC) } 8 \\
\text { IL) }\end{array}$ & 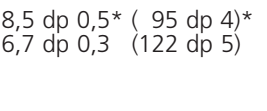 & $\begin{array}{l}\text { IC) } 52 \mathrm{dp} 3^{*} \\
\text { IL) } 37 \mathrm{dp} 2\end{array}$ & 0-60min \\
\hline Dolezal et al. ${ }^{6}$ & $\begin{array}{l}18 \mathrm{H}(9 \mathrm{~T}, 9 \mathrm{DT}) \\
(21 \mathrm{dp} 2)\end{array}$ & $\begin{array}{l}\text { Ex: Leg press, l: } 3 \mathrm{~min} \\
8 x 6 \mathrm{RM} \\
\text { Excêntrico (4s) }\end{array}$ & & -- & $\begin{array}{l}\mathrm{DT} / \mathrm{T}) \mathrm{ND} \\
\text { Elevado até } 48 \mathrm{~h}\end{array}$ & $\begin{array}{l}\text { TMR 24, 48, } \\
72 \mathrm{~h}\end{array}$ \\
\hline Beckham \& Earnest ${ }^{27}$ & $\begin{array}{l}20(12 \mathrm{H}, 8 \mathrm{M}) \mathrm{T} \\
(18-45)\end{array}$ & $\begin{array}{l}\text { Ex: } 5 \\
\text { IB) } \mathrm{HBL} 1,4 \mathrm{~kg}, \mathrm{HBC} \\
1,1 \mathrm{~kg}(\mathrm{H} / \mathrm{M}) \\
\text { IA) } \mathrm{HBL} 10,5 \mathrm{~kg}(\mathrm{H}), 5,9 \mathrm{~kg}(\mathrm{M}) \\
\mathrm{HBC} 4,5 \mathrm{~kg}(\mathrm{H}), 2,3 \mathrm{~kg}(\mathrm{M})\end{array}$ & IB) & $\begin{array}{l}H: 5,0 \text { dp } 0,8^{*} \\
\left.M: 3,6 \text { dp } 0,5^{*} \neq \mid A\right) \\
H: 6,2 \text { dp } 1,0 \\
M: 4,0 \text { dp } 0,5\end{array}$ & --- & --- \\
\hline Thornton \& Potteiger ${ }^{7}$ & $14 \mathrm{MT}$ (27dp 5) & $\begin{array}{l}\text { Ex: } 9, \text { I: } 60 \text { s } \\
\text { IA) } 2 \times 8 r, 85 \% \text { 8RM, DS: } 23 \\
\text { IB) } 2 \times 15 r, 45 \% 8 R M, D S: 26\end{array}$ & $\begin{array}{l}\text { IA) } 2 \\
\text { IB) } 2\end{array}$ & $\begin{array}{l}2,77(64 \mathrm{dp} 7) \\
2,75(72 \mathrm{dp} 7)\end{array}$ & $\begin{array}{l}\text { I: } 11 \mathrm{dp} 2^{*} \\
\text { L: } 6 \mathrm{dp} 1\end{array}$ & $\begin{array}{l}0-20 \mathrm{~min} \\
45-60 \mathrm{~min} \\
105-120 \mathrm{~min}\end{array}$ \\
\hline Schuenke et al. ${ }^{24}$ & 7HT (19-26) & $\begin{array}{l}\text { C, Ex: } 4,1: 120 \mathrm{~s} \\
4 \times 8-12 \text { RM }\end{array}$ & & -- & $\begin{array}{l}\mathrm{ND} \\
\text { Elevado até } 38 \mathrm{~h}\end{array}$ & $\begin{array}{l}0,14,19,24 \\
38,43,48 h\end{array}$ \\
\hline Hunter et al. ${ }^{28}$ & 7HT (24 dp 4) & $\begin{array}{l}\text { Ex: 10, DS: } 29 \\
\text { Tradicional (T): } 2 \times 8 r, 65 \% \\
1 \text { RM, I: } 60 \mathrm{~s} \\
\text { Super lento (SL): } 1 \times 8 \mathrm{r}(10 \mathrm{~s} \\
\text { cond } 5 \mathrm{~s} \text { exc), } 25 \% 1 \mathrm{RM}\end{array}$ & $\begin{array}{l}\mathrm{T}: \\
\mathrm{SL}:\end{array}$ & $\begin{array}{l}3,9(114 \mathrm{dp} 10)^{*} \\
2,5(\mathrm{~T}-\mathrm{dp} \mathrm{13)}\end{array}$ & $\begin{array}{l}\text { T: } 41 \mathrm{dp} 10^{*} \\
\text { SL: } 34 \mathrm{dp} 8\end{array}$ & $0-15 \min$ \\
\hline Phillips \& Ziuraitis ${ }^{45}$ & $\begin{array}{l}10(5 \mathrm{H}, 5 \mathrm{M}) \mathrm{T} \\
(73 \mathrm{dp} 6)\end{array}$ & $\begin{array}{l}\text { Ex: } 8, \mathrm{l}: 120 \mathrm{~s} \\
1 \times 15 \mathrm{RM}\end{array}$ & $\begin{array}{l}\mathrm{H}: \\
\mathrm{M}:\end{array}$ & $\begin{array}{l}3,5 \mathrm{dp} 0,6(84,2 \mathrm{dp} 14,6) \\
2,9 \mathrm{dp} \mathrm{0,7}(69,7 \mathrm{dp} 17,4)\end{array}$ & --- & --_- \\
\hline Morgan et al..$^{33}$ & $\begin{array}{l}15(8 \mathrm{H}, 7 \mathrm{M}) \mathrm{T} \\
(20-29)\end{array}$ & $\begin{array}{l}\text { Ex: } 8,1: 45 \mathrm{~s} \\
\text { IB) } 2 \times 15 \mathrm{r}, 85 \% 8 \mathrm{RM} \\
\text { IA) } 2 \times 8 \mathrm{RM}\end{array}$ & IB) & $\begin{array}{l}\mathrm{H}: 2,8 \mathrm{dp} 1,5(58 \mathrm{dp} 31) \\
\text { M: 2,5 dp 0,7 (58 dp 17) } \\
\mathrm{H}: 2,7 \mathrm{dp} 0,8(51 \mathrm{dp} 15) \\
\text { M: 2,3 dp 0,8 (42 dp 15) }\end{array}$ & -- & -- \\
\hline Haddock \& Wilkin ${ }^{47}$ & $15 \mathrm{MT}$ & $\begin{array}{l}\text { Ex: } 9 \\
1 \text { serie (1): } 1 \times 8 \mathrm{RM} \\
3 \text { series (3): } 3 \times 8 \mathrm{RM}\end{array}$ & 1) & $\begin{array}{c}56 \mathrm{dp} 3 \\
158 \mathrm{dp} 10^{*}\end{array}$ & $\begin{array}{l}\text { 1) } 22 d p 5 \\
\text { 3) } 22 d p 2\end{array}$ & $0-120 \mathrm{~min}$ \\
\hline Henley et al. ${ }^{46}$ & $\begin{array}{l}8(2 \mathrm{H}, 6 \mathrm{M}) \mathrm{T} \\
(23 \mathrm{dp} 4)\end{array}$ & $\begin{array}{l}\text { Ex: } 10, \text { I: } 120 \mathrm{~s} \\
1 \text { serie }(1): 1 \times 10 \mathrm{RM} \\
3 \text { series (3): } 3 \times 10 \mathrm{RM}\end{array}$ & 1) & $\begin{array}{l}75 \mathrm{dp} 28 \\
208 \mathrm{dp} 83^{*}\end{array}$ & $\begin{array}{l}\text { 1) } 36 \mathrm{dp} 20 \\
\text { 3) } 52 \mathrm{dp} 22\end{array}$ & $\begin{array}{l}0-30,45-60, \\
75-90, \\
105-120 \mathrm{~min}\end{array}$ \\
\hline Jamurtas et al. ${ }^{20}$ & $10 \mathrm{H}(22 \mathrm{dp} 2)$ & $\begin{array}{l}\text { Ex: } 10,70-75 \% 1 \mathrm{RM} \\
4 \times 8-12 \mathrm{RM}\end{array}$ & & -- & $\begin{array}{l}\mathrm{ND} \\
\text { Elevado até } 24 \mathrm{~h}\end{array}$ & $\begin{array}{l}0,10,24,48, \\
72 \mathrm{~h}\end{array}$ \\
\hline Phillips \& Ziuraitis ${ }^{44}$ & $\begin{array}{l}12(6 \mathrm{H}, 6 \mathrm{M}) \mathrm{T} \\
(27 \mathrm{dp} 4)\end{array}$ & $\begin{array}{l}\text { Ex: 8, DS: } 24, \mathrm{I}: 120 \mathrm{~s} \\
1 \times 15 \text { RM }\end{array}$ & $\begin{array}{l}\text { H) } \\
\text { M) }\end{array}$ & $\begin{array}{l}5,6 d p 0,7(135 d p 16) \\
3,4 d p 0,5\left(\begin{array}{rl}13 \\
82 & 11\end{array}\right)\end{array}$ & --- & --- \\
\hline $\operatorname{Scott}^{31}$ & $\begin{array}{l}11(5 \mathrm{H}, 6 \mathrm{M}) \mathrm{T} \\
(\mathrm{H}: 26 \mathrm{dp} 8 \\
\mathrm{M}: 25 \mathrm{dp} 7)\end{array}$ & $\begin{array}{l}\text { Ex: } 3, \text { I: } 2,5 \mathrm{~min} \\
\text { IB) } 2 \times 60 \% 1 \mathrm{RM} \\
\text { IA) } 2 \times 80 \% 1 \mathrm{RM}\end{array}$ & $\begin{array}{l}\text { IB) } 5 \\
\text { IA) } 3\end{array}$ & $\begin{array}{l}55,6^{* *} \\
34,4^{* *}\end{array}$ & --_ & -_-_ \\
\hline Ormsbee et al. ${ }^{21}$ & $\begin{array}{l}8 \mathrm{M} \\
(21-27)\end{array}$ & $\begin{array}{l}\text { Ex: 6, I: } 90 \text { s, } 3 \times 10 \\
85 \% 10 \text { RM, DS: } 40 \mathrm{~min}\end{array}$ & & --- & $104,4 \mathrm{dp} 6,0 \mathrm{kcal} . \mathrm{h}^{-1}$ & $45 \mathrm{~min}$ \\
\hline
\end{tabular}

$H=$ homens; $M=$ mulheres; $T=$ treinados; $D T=$ destreinados; $C=$ circuito; $E x=n^{\circ}$ exercícios; $D S=$ duração da sessão (min); l= intervalo entre séries; $R M=$ repetições máximas; $\mathrm{r}=$ repetiçōes; $\mathrm{HBC}=$ halter de barra curta; $\mathrm{HBL}=$ halter de barra longa; $\mathrm{IA}=$ intensidade alta; $\mathrm{IB}=$ intensidade baixa; $\mathrm{DAC}=$ doença arterial coronariana; $\mathrm{ND}=$ não disponivel * diferença significante entre grupos; diferença significante entre homens e mulheres; $\mathrm{dp}=$ desvio-padrão; **soma dos valores reportados pelo autor. 